\title{
LITERATURA ANTROPOLÓGICA EN CHILE: UNA MANIFESTACIÓN DE LITERATURA INTERCULTURAL ${ }^{1}$
}

\author{
Pilar Valenzuela Rettig \\ Universidad Autónoma de Chile, Temuco, Chile \\ valenzuela.pilar@gmail.com
}

\section{RESUMEN / ABSTRACT}

La literatura antropológica en Chile corresponde a una literatura interdisciplinaria e intercultural, escrita por antropólogos y arqueólogos chilenos contemporáneos, que buscan en ella una nueva forma de comunicar la experiencia del trabajo de campo y el conocimiento antropológico. En este artículo se presentan las principales conclusiones de una investigación cuyo objetivo fue definir y caracterizar la literatura antropológica como un tipo de literatura intercultural.

Palabras clave: literatura antropológica, literatura intercultural, interculturalidad, interdisciplinariedad.

\section{Anthropological Literature in Chile: The Manifestation OF INTERCULTURAL LITERATURE}

Anthropological literature in Chile is an intercultural and interdisciplinary literature, written by contemporary Chilean anthropologists and archaeologists. They are looking for a new way of communicating their field work and anthropological knowledge. This article presents the main conclusions of a research whose objective was to define and characterize anthropological literature as an intercultural literature.

KEYWORDS: anthropological literature, intercultural literature, intercultural, interdisciplinarity.

Recepción: 01/05/2017

Aprobación: 21/07/2017

1 El presente trabajo se enmarca en el Proyecto FONDECYT Postdoctoral, №3140616, "Literatura antropológica en Chile: una literatura intercultural", que contó con el patrocinio del Dr. Hugo Carrasco y la Universidad de La Frontera. 


\section{LITERATURA ANTROPOLÓGICA E INTERCULTURAL EN CHILE}

La literatura antropológica en Chile surge, como teoría literaria, en el año 2010, con la publicación del artículo "Literatura antropológica chilena: fundamentos" de Iván Carrasco en Estudios Filológicos ${ }^{2}$. En este trabajo, Carrasco determina la existencia de la literatura antropológica a partir de la determinación de un tipo de texto no descrito desde la perspectiva de la teoría, de la crítica ni la historia literaria: un conjunto de textos escritos por antropólogos y arqueólogos, que corresponden a una nueva clase de literatura fronteriza, caracterizada por la mutación disciplinaria ${ }^{3}$ (Carrasco, Literatura antropológica 9). Define el autor la literatura antropológica en Chile como:

... un tipo de discurso que conlleva las condiciones suficientes para ser leído simultánea o alternativamente como texto literario y/o como etnográfico... un conjunto de textos literarios de carácter principalmente etnográfico, escritos por antropólogos y arqueólogos contemporáneos, textos mediacionales en busca de nuevas experiencias y modos de conocimiento, como la intuición creadora del poeta o novelista (...) [una literatura que] ha surgido en medio de la escritura antropológica y puede considerarse un sector de la literatura chilena por su parcial analogía temática y estilística con la textualidad literaria moderna y postmoderna, pero al mismo tiempo no ha perdido sus cualidades etnográficas y etnológicas (13-14).

Carrasco sostiene que esta literatura se sustenta tanto en la interdisciplinariedad, como en la interculturalidad. Definiendo éstas como estrategias estructurantes de la literatura de fines del siglo XX y comienzos del XXI, que hacen posible la modificación, ampliación y transformación de los modos de configurar y proponer la lectura de los textos, abarcando las posibilidades inter y transdisciplinarias.

2 Este artículo formó parte del Proyecto FONDECYT Regular 2010, №1100344, "Literatura antropológica en Chile".

3 La mutación disciplinaria corresponde a la modificación de las reglas, modalidades, materias y procedimientos de conformación de textos de una disciplina artística, científica o filosófica, provocada por el traslado desde otra u otras disciplinas de la misma o distinta condición; dando por resultado la confusión de campos disciplinarios, géneros y tipos discursivos. Por tanto, el tipo de texto producido se caracteriza por la heterogeneidad, confluencia o mezcla de géneros, contenidos y procedimientos de disciplinas distintas que coexisten en él de diferentes modos (Carrasco, Interdisciplinariedad). 
Dentro de las cualidades propias de la disciplina antropológica, el crítico considera que esta textualidad ha mantenido, dentro del proceso de mutación disciplinaria, las siguientes características propias de la antropología: la reiteración de temáticas como la del otro diverso, la importancia del viaje como modo de encontrar experiencias y conocimiento, la descripción detallada de situaciones de contacto intercultural e interétnico, diversos indicios de la formación profesional de los autores-narradores y la perspectiva o punto de vista del antropólogo (14). Por el otro lado, sostiene que lo literario de la textualidad se refiere principalmente a: "la percepción o concepción de la literatura como experiencia estética de las escrituras y las lecturas, y en cuanto modo de conocimiento de la sociedad, las culturas y las formas de ser y de vivir, [y] en grado menor como testimonio autobiográfico del investigador" (14).

Con este artículo del 2010, Carrasco inicia el proceso de canonización de la textualidad dentro del campo literario, trasladando el objeto de estudio que hasta la fecha se mantenía dentro del campo antropológico bajo la denominación de "antropología poética" o "antropología literaria"4. Esto, debido a que el origen de la textualidad se ubica, efectivamente, dentro del campo de la antropología y ciencias sociales chilenas. Miguel Alvarado (Introducción a la antropología poética), principal investigador de esta textualidad dentro del campo antropológico, determina que surge dentro del campo antropológico y que, con una intención antropológica, se utiliza el lenguaje literario como recurso expresivo. Es enfático en sostener que esta textualidad surge como un subgénero antropológico, donde la literatura adquiere un carácter instrumental, sosteniendo que "es producto de una transformación de la discursividad antropológica que descubre sus límites y asume la potencialidad de la literatura para ampliar sus posibilidades expresivas" (La antropología poética chilena 336).

4 Miguel Alvarado ha postulado la denominación de "antropología literaria", estableciendo un conjunto textual que, al igual que la "antropología poética", inserta en el desarrollo de la textualidad antropológica. Las diferencia en medida en que la "antropología literaria" corresponde a una textualidad latinoamericana, no sólo chilena, que presenta una intencionalidad y un desarrollo más teórico que la "antropología poética", por cuanto se propone generar conocimiento tras la superación de la distinción sujeto-objeto, mediante una propuesta de acceder a la diversidad a través de una escritura que se constituye a partir de las estrategias de literaturización y analogía estética con la literatura (Valenzuela, Literatura antropológica 163). 
Alvarado determina como precursores de la textualidad a los antropólogos Andrés Recasens y Carlos Piña ${ }^{5}$; mientras que a Juan Carlos Olivares lo instaura como el iniciador de la corriente con la publicación en 1995 de El umbral roto. Escritos en antropología poética. Efectivamente, es en el texto de Olivares donde aparece por primera vez una propuesta metatextual de la antropología poética, provocando una recepción que inicia y posibilita el tránsito desde la recepción antropológica hacia la literaria ${ }^{6}$.

El carácter intercultural de la literatura antropológica se establece en primera instancia por Carrasco en su artículo publicado el 2010, y se profundiza y justifica, a nivel teórico, en el artículo "Literatura antropológica en Chile: ¿Una literatura intercultural?" (Valenzuela) publicado el año 2015 por Acta Literaria. En este artículo, se delimita y aclara el concepto de interculturalidad, en base a una concepción histórica y dinámica de "cultura/s", que considera que estas corresponden a procesos cuyas fronteras se encuentran en continuo tránsito, por lo que no pueden ser consideradas monotradicionales y que, así

5 Andrés Recansens es considerado por Alvarado (Introducción a la antropología poética) como precursor de la corriente a partir de la creación del poema "Balada para niño y perro". Poema que surge de una experiencia de trabajo etnográfico que realizó como profesor de la Universidad de Chile a un grupo de estudiantes de antropología en 1975. Experiencia que relata en su ponencia “¿Antropología poética o poesía antropológica?”, presentada en el Primer Encuentro Nacional de Antropología Poética, en 1988, ciudad de Ancud (sin publicar). El poema, expresa su autor, surge como el discurso adecuado para comunicar la situación observada de un niño vago enfermo sin más compañía que un perro. Por otra parte, Carlos Piña es considerado por Alvarado como precursor de la corriente textual, debido a la publicación, en 1987, del libro Crónicas de la otra ciudad, donde presenta narraciones literarias creadas a partir del trabajo etnográfico.

Al respecto, cabe destacar la definición de antropología poética que propone Daniel Quiroz en el libro de Olivares: "Una etnografía poética no es sino crear la descripción del encuentro con la imaginación y rigurosidad que nos exige la poesía. La poesía nos permite transmitir las emociones y sensaciones que nos provoca el encuentro entográfico" (16). Para conocer la recepción del Umbral roto y su tránsito desde la antropología poética hacia la literatura antropológica, confróntese Valenzuela (Entre antropología).

La definición de interculturalidad en este artículo se constituye en base a un marco teórico congruente con el artículo "Literatura intercultural chilena: proyectos actuales" de Iván Carrasco, debido a que este es el único estudio que abarca en conjunto las manifestaciones interculturales chilenas, proponiendo una lectura teórica que, hasta el momento, no ha sido refutada ni corregida por otro investigador (a nivel de teoría general, que engloba diversas manifestaciones de literaturas interculturales en Chile). Además de establecer un marco de coherencia intertextual con la propuesta teórica de Carrasco en "Literatura antropológica chilena: fundamentos". 
mismo, se hallan en constante proceso de construcción, al componerse en la permanente interacción de los seres humanos que conforman una comunidad (165). A partir de estas aseveraciones, se entiende por "interculturalidad" una situación concreta que se manifiesta en las "relaciones interculturales", donde debe darse el reconocimiento mutuo, el intercambio de saberes y experiencias, y la convivencia social; relaciones que se plasman en una "comunicación intercultural interpersonal" que requiere de una competencia comunicativa y un cierto conocimiento de la otra cultura (165-6).

En este trabajo se reconoce que la literatura antropológica puede considerarse una literatura intercultural, por cuanto: en primer lugar, se condice con la definición de "interculturalidad literaria" que presenta Carrasco ${ }^{8}$. Corresponde a este tipo de textualidad en la medida en que se crea como parte de un proceso de construcción y redefinición textual e identitaria a partir de una situación de interculturalidad concreta (sociocultural): en este caso, entre los profesionales (antropólogos y arqueólogos que presentan una cultura social y disciplinaria particular) en contacto intercultural con las personas que forman parte de la "otra cultura" en estudio. En segunda medida, se sostiene que la literatura antropológica puede considerarse una literatura intercultural por presentar las características esenciales ${ }^{9}$ de los proyectos de literatura intercultural presentados por Carrasco en el 2005: 1) dar cuenta de una situación intercultural, 2) la doble codificación, 3) la intertextualidad y 4) una enunciación sincrética, intercultural o heterogénea; características que presentan los proyectos de literatura intercultural que Carrasco delimita al $2005^{10}$ y que se identifican como elementos presentes en la teoría de la literatura antropológica en Chile ${ }^{11}$.

8 Para Carrasco, la "interculturalidad literaria" corresponde a "la relación entre etnias, culturas, lenguas y dialectos producida en textos reconocidos como literarios por la institución literaria chilena, que se modifican y transforman en la compleja situación de interacción o reciprocidad en que se hallan. .." (Carrasco, Literatura intercultural 68).

9 Se consideran "características esenciales" por determinarse como las características que Carrasco presenta en el artículo del 2005 para definir las literaturas interculturales.

10 Los proyectos de literatura intercultural que Carrasco determina al 2005 corresponden a: la poesía etnocultural, donde se encuentran los proyectos de escritura de los escritores mestizos de origen europeo, el de los mapuches y de los chilotes; la literatura del exilio, y la de los inmigrantes.

11 Tómese como principal referencia con respecto a la "teoría de la literatura antropológica en Chile" el artículo de Carrasco del 2010 y la tesis doctoral de Pilar Valenzuela (El diario de viaje). 


\section{INTERCULTURALIDAD EN LA LITERATURA ANTROPOLÓGICA}

Si bien las características de la literatura intercultural son identificadas y analizadas desde la perspectiva teórica en la literatura antropológica en el trabajo de Valenzuela (Literatura antropológica), se requería justificar estas y el carácter intercultural de la textualidad a partir del análisis del corpus: estudio que se presenta a continuación.

El corpus de estudio fue compuesto por 39 textos $^{12}$. Estos corresponden al corpus de la literatura antropológica al año 2015, siendo delimitados por la autoría de antropólogos o arqueólogos contemporáneos (desde el año $1975^{13}$ en adelante) y presentar características que correspondan a saberes, o códigos, antropológicos y literarios. El corpus quedó conformado por 22 libros, siendo dos de ellos compilaciones de textos de antropólogos, de los cuales se analizaron 18 textos (siendo excluidos algunos textos de estas compilaciones por no manifestar un carácter interdisciplinario; es decir, por no presentar acercamiento hacia la literatura). Por tanto, se analizaron textos de 21 autores: Andrés Recasens, Carlos Piña, Daniel Quiroz, Leonor Adán, Patricio Toledo, Francisco Gallardo, Alfredo Gahona, Flora Vilches, Yuri Jeria, Juan Carlos Olivares, Rafael Prieto, Mauricio Massone, Mauricio Osorio, Alexis Rojas, Dagoberto Ramírez, Sonia Montecino, Clemente Riedemann, Yanko González, Ivonne Valenzuela, Claudio Mercado y Pedro Mege. Destacando dentro de los libros: El umbral roto. Escritos en antropología poética (1995) de Juan Carlos Olivares, Atacameños del siglo XX. Registro fotográfico y etnográfico (1998) de Ivonne Valenzuela, y Metales Pesados (1998) de Yanko González Cangas, debido a que han sido analizados de forma particular por su valor estético y metadiscursivo, presentando ponencias y artículos referidos a ellos ${ }^{14}$.

12 Ver "Anexo: corpus de estudio".

13 Año en que Recasens crea el poema "Balada para niño y perro".

14 Entre los estudios de estos textos destacan los artículos sobre los textos de Ivonne Valenzuela (Poesía antropológica de Carrasco y Fotografía de Valenzuela), sobre El umbral roto (Entre antropología de Valenzuela) y de Metales Pesados (Interdisciplinariedad de Valenzuela). 
El análisis de los textos se realizó a partir de una metodología semiótica ${ }^{15}$ y considerando los siguientes objetivos: 1) identificar y analizar los marcos discursivos y los paratextos en relación con la interculturalidad e interdisciplinariedad, 2) identificar y especificar las estrategias discursivas propias de la literatura intercultural presente en los textos y 3) analizar la relación sujeto-informante presente en los textos a partir de los conceptos de otredad e interculturalidad, siendo estos enmarcados en el desarrollo del objetivo general de definir y caracterizar la literatura antropológica como un tipo de literatura intercultural en Chile donde el sujeto antropólogo/ arqueólogo presenta una mirada intercultural del trabajo de campo que supera la otredad, dando pie a una experiencia intercultural que requiere de estrategias interdisciplinarias para ser comunicada.

\section{INTERCULTURALIDAD E INTERDISCIPLINARIEDAD}

A partir de la identificación y análisis de los marcos discursivos y paratextos en relación con interdisciplinariedad e interculturalidad, se concluye que la gran mayoría de los textos presenta estos elementos ${ }^{16}$, concluyendo que interculturalidad e interdisciplinariedad ${ }^{17}$ se manifiestan, a través de los marcos discursivos y paratextos, como características constituyentes de una propuesta de desarrollo de la antropología. Esta propuesta se funda en la crítica hacia esta disciplina y ciencias sociales tradicionales, para presentarse

15 A grandes rasgos, la metodología del estudio corresponde a una lectura semiótica, basada en la teoría semiótica de Umberto Eco, principalmente en la lectura semiótica centrada en la intención del texto (Los límites de la interpretación; Tratado; Seis paseos).

16 En cuanto a la identificación de estos elementos de interculturalidad e interdisciplinariedad en el corpus, concluimos que la mitad de los textos $(51,2 \%)$ presenta marcos discursivos y paratextos que permiten analizarlos en relación con estos conceptos; un porcentaje menor $(41 \%)$ presenta parcialmente estos elementos de análisis y tan solo 3 textos no los presentan $(7,6 \%)$.

17 El concepto de "interdisciplinariedad" se aborda desde la revisión bibliográfica centrada en la situación de América Latina y, principalmente, la teoría desarrollada sobre antropología poética y literaria. A partir de esta investigación se identifica la necesidad de ahondar en la teoría de la complejidad como posibilidad de ampliar el análisis de la textualidad; así como la distinción entre modernidad y posmodernidad en relación con la manifestación de textos interdisciplinares; temas que quedan presentes como posibilidad de tratar en investigaciones futuras. 
como una escritura novedosa, experimental y personalista. Escritura que reconozca el valor de las personas involucradas, tanto investigador como informante, en su particularidad; junto al reconocimiento de la importancia de la experiencia del contacto intercultural entre ambos, como experiencia de vida y forma de conocimiento: situación de interacción que se excluye de la escritura antropológica tradicional, al igual que el autor como sujeto ${ }^{18}$. Por otro lado, la interdisciplinariedad se presenta como propuesta escritural ante la necesidad expresiva que nace de la urgencia de un modo de comunicación que supere los límites impuestos por el discurso científico. Se trata, a través de la amalgama de elementos antropológicos y literarios, de comunicar una situación de trabajo de campo o investigación etnográfica sobre otra cultura, que impacta en la persona del antropólogo o arqueólogo. Impacto que obedece a una disposición integral del sujeto, quien vive la experiencia de contacto y conocimiento del otro involucrándose como ser humano, más allá del rol de científico.

Entre los textos más relevantes en cuanto a marcos discursivos y paratextos en relación con los elementos de interculturalidad e interdisciplinariedad, encontramos Arqueología y poesía (1997) de Francisco Gallardo. En este, se presenta el paratexto introductorio de Juan Carlos Rodríguez, que avala la innovación escritural de Gallardo como una necesidad actual de personalizar el discurso científico:

La liturgia de la academia para exaltar a su salvaje exilió la fantasía, el juego, la emoción, el sueño, la noche y el deseo del relato antropológico, enhebrando su construcción en la lógica de la razón, como si la vida, la condenada vida de los antropólogos, pudiese sustraerse al despertar de las propias emociones cada vez que se es asediado por los fragmentos de un pasado, por el rostro presentificado de nuestros contemporáneos, la provocación de los objetos salidos de una excavación o de una casa vestida para todas las ocasiones. Nos ha costado sacudirnos del pánico al vacío y la demanda perenne del ajuste cultural y epistemológico de nuestra época (8).

18 Se presenta esta lectura de la literatura antropológica como literatura intercultural, desde el personalismo, en la ponencia titulada "Investigar desde y por la persona: literatura antropológica", presentada en el III Congreso Iberoamericano de Personalismo, organizado por la Universidad Técnica Particular de Loja y la Asociación Iberoamericana de Personalismo, en la ciudad de Loja, Ecuador (julio, 2015). 
Rodríguez, en este paratexto, reacciona ante enunciados metatextuales en los cuales Gallardo critica a la ciencia, validando la unión entre ciencia y poesía, imaginación y conocimiento, y la libertad, en base a la certeza de la poética como parte de un lenguaje nuevo. Destacamos, dentro de los enunciados de Gallardo, el siguiente párrafo, donde reflexiona sobre la relación entre ciencia y poesía:

Ninguna ciencia que se considere madura a sí misma, puede pretender renunciar a la poesía que emerge hacia la superficie en los lindes de su práctica, negarla sólo sería un acto de impertinencia hacia la misma realidad que nos consume. La poesía y la ciencia están sometidas a la creación, y nada en este mundo que pertenezca al reino de la novedad está libre de ese hecho insondable que se consume como un arrebato de la imaginación. La imagen poética es una forma de comunicar nuestro entusiasmo ante los hechos de indagación y conocimiento. Sin embargo, el proceso es insondable, pues aunque no sabemos por qué, sabemos que sólo algunos hechos nos conciernen poéticamente. Desde la profundidad oceánica de este misterio ascienden las metáforas y sus imágenes, provocando en el alma (que es algo como el primer aire que respiramos) del que ordena y analiza un sentimiento de libertad infinita, porque la poesía es siempre trasgresión del lenguaje, emergencia de lenguaje nuevo (18).

Con respecto a la interculturalidad, destaca el libro De todo el universo entero (1997) de Claudio Mercado y Luis Galdames (Quilama). En este, Mercado, arqueólogo y músico, comparte autoría con Galdames, un pescador y "chino" que participa en las festividades religiosas en la zona circundante a La Serena. Ambos autores participan en la conformación de un texto donde se hacen presente las dos voces: la del que aprende (el arqueólogo que tiene interés en aprender y participar de los rituales) y del que enseña (el chino que logra la inserción y aprendizaje del santiaguino en estas ceremonias), dando lugar a une relación intercultural que José Pérez de Arce, en el "Prólogo" califica mediante la metáfora del "puente":

Tender puentes entre universos, obsesión de Claudio, alcanza en este libro un gran logro. Entre él, urbano, con su herencia occidental, y Quilama, el pescador, se tiende un puente mágico: a ambos los une el estudio, a Claudio la arqueología, la música, la musicología, a Quilama la Biblia. Quilama habita su fe milenaria con la tremenda experiencia de pescador, Claudio habita el complejo sistema científico occidental. 
Pero en ambos está abierta la puerta a la percepción, del arte. Es allí donde ambos se cuelan a otros mundos y nace este libro. Este libro es obra de Claudio, porque él sabe cómo escribir sus verdades, pero también es de Quilama, que habla las mismas verdades con otro idioma. Y ése es el gran mérito de este libro: ser el perfecto puente entre dos universos con la misma ambición de lo inexpresable (10).

\section{PRESENCIA DE ESTRATEGIAS DISCURSIVAS INTERCULTURALES}

En cuanto al objetivo de identificar y especificar las estrategias discursivas propias de la literatura intercultural presente en los textos, se identificaron, en primera instancia, las estrategias discursivas de la literatura intercultural en Chile, como ya se expuso. Estas corresponden a: 1) dar cuenta de una situación intercultural, 2) doble codificación, 3) intertextualidad, 4) enunciación sincrética, intercultural o heterogénea, siendo presentadas en un artículo anterior (Valenzuela, Literatura antropológica).

Con respecto a la estrategia de "dar cuenta de una situación intercultural", la particularidad de la literatura antropológica corresponde a que la situación (como macroestructura semántica) se determina por el contexto del viaje y/o trabajo antropológico, y su enunciación se caracteriza por la interdisciplinariedad (entre el discurso antropológico y literario). Del corpus en estudio, la mayoría presenta esta estrategia ${ }^{19}$, dentro de los cuales destaca Sueño con menguante (1999) de Sonia Montecino. Esta es una novela escrita y narrada por una antropóloga que comparte una historia y el espacio de proyección de la voz narrativa, junto a Carmela, una machi. Corresponde a una situación intercultural que se genera a partir del viaje de la joven estudiante de antropología al sur, junto a Florencia Huenuman (mapuche que vive en la capital) para conocer a su familia y el mundo mapuche: en este viaje conoce a la machi, con quien establece un vínculo de amistad y enseñanza. La situación de interculturalidad se justifica, principalmente, en cuanto se presenta una situación de interculturalidad sociocultural entre los personajes de la antropóloga y la autora, situación que también se puede hipotetizar como una experiencia real entre ambas mujeres (como personas

19 Del corpus, un $48,7 \%$ presenta la estrategia de manera clara y destacadas, un $15 \%$ de forma parcial y tan solo un $12 \%$, correspondiente a 5 textos, no la presenta. 
reales, no personajes), que genera la creación de este texto que se construye a partir de la temática antropológica, pero cuya construcción se acerca más a la novela que al diario o cuaderno de campo.

En relación con la estrategia de "doble codificación", esta literatura se distingue por presentarla tanto a nivel lingüístico (como la literatura etnocultural), como a nivel disciplinar, por constituirse a partir de códigos antropológicos y literarios ${ }^{20}$. Como ejemplo de esta estrategia podemos destacar el poemario Karra Maw'n (1984) de Clemente Riedemann, donde se desarrolla la doble codificación "disciplinar": por cuanto la temática es sobre la historia cultural del sur de Chile, a partir de las relaciones entre las culturas mapuches, españolas y alemanas, y el lenguaje es poético; y la doble codificación "lingüística", en la medida en que se plasman enunciados en los idiomas de esta cultura. Como ejemplo de doble codificación en Karra $M a w^{\prime} n$ encontramos los siguientes versos, que hacen referencia al encuentro entre españoles y mapuches:

Los indios desconfiaron de Chaw-Ngëchén

EL SER DIVINO

Cuando vieron muchos hierros

y caballos

¡WINKA! -dijieron,

¡KIÑE PATAKA PIKUNPÜLE! -dijieron,

y fueron a consultar al guardador de secretos

y leyendas:

“iNIELOL DUGUTUM TRALKAN!”

$\mathrm{Y}$ sintieron temor.

(La maldad del wekufe 16)

La estrategia de "intertextualidad" 21 se relaciona con la de "intertextualidad transliteraria" que presenta la poesía etnocultural. No obstante, por ser una

$20 \quad$ Más de la mitad de los textos que conforman el corpus presenta esta estrategia de manera destacada $(69,2 \%)$, once textos la contienen parcialmente y tan solo un texto (Gracias por favor concedido de Ivonne Valenzuela) no la presenta.

21 En proporción, esta estrategia junto con la de enunciación sincrética, intercultural o heterogénea se presentan ya sea completa o parcialmente, en más de la mitad del corpus (un $46,1 \%$ completamente, $17,9 \%$ de forma parcial y $35,8 \%$ de los textos no presenta esta característica). 
textualidad interdisciplinaria, no puede distinguirse como "transliteraria", porque la intertextualidad se presenta hacia lo transliterario como hacia lo literario. Uno de los textos que destaca por su carácter de intertextualidad es El umbral roto. Escritos en antropología poética (1995) de Juan Carlos Olivares, donde aparecen citas y referencias literarias a El reino de este mundo de Carpentier y Cien años de soledad de García Márquez, por ejemplo, y referencias transliterarias a Lévi-Strauss, Focault, Guside y Castaneda, entre otras.

Por último, la estrategia de "enunciación sincrética, intercultural o heterogénea" 22 se caracteriza por la presencia de las voces del antropólogo y de los informantes (los “otros") que también se explayan como narradores o dialogantes. Así mismo, se cumple cuando el enunciante se presenta como un "sujeto ambiguo", que se debate entre dos ciencias y dos culturas, yendo y viniendo de una a otra, por ejemplo en Metales pesados (1998) de Yanko González o Antiguas historias del valle Simpson (2014) de Mauricio Osorio; experimentando ser otro, por ejemplo en Atacameños del siglo XX. Registro fotográfico y etnográfico (1998) de Ivonne Valenzuela; y experimentando una situación de inestabilidad que cuestiona su identidad, no solo cultural, sino también profesional, por cuanto ésta puede leerse como una subcultura: la de la literatura antropológica dentro de la cultura científica.

\section{RELACIÓN SUJETO-INFORMANTE}

Con respecto al objetivo de "analizar la relación sujeto-informante presente en los textos a partir de los conceptos de otredad e interculturalidad", se partió por definir el concepto de otredad como el objeto de estudio de la antropología, que no se refiere a una simple diferenciación, sino que se relaciona con la experiencia de los extraños. Al respecto, Marc Augé en El sentido de los otros pone en evidencia la situación que denomina "paradoja fundacional de la antropología": el hecho de que al antropólogo se le pida que entienda otras culturas desde el interior y el exterior al mismo tiempo, distanciada en la medida en que se reconoce la alteridad entre observador y lo observado, y participativa en cuanto necesita estar dentro de la sociedad para contar con

22 Dentro del análisis de esta estrategia, los resultados de presencia proporcionales son iguales a la de la categoría anterior. Ver nota al pie $n^{\circ} 18$. 
la información necesaria para la interpretación de la cultura. En contraste, el carácter intercultural que sostenemos que presentan los textos, considera que la mirada, perspectiva y/o relación del sujeto con su objeto de estudio supera la otredad, en la medida en que supera la paradoja exterioridad-interioridad a través de la interculturalidad. Es a través de esta que el sujeto se contacta con el otro, reconociéndose a partir de sus semejanzas y diferencias, buscando los puntos de encuentro, aprendiendo del otro y/o cuestionándose sus propios parámetros culturales.

Del análisis general del corpus podemos afirmar que la mayoría de los textos presenta una relación sujeto-informante donde se demuestra la superación de la otreadad por la interculturalidad ${ }^{23}$. Consideramos que este segmento presenta mayor cantidad de textos con ausencia de esta característica (la relación sujeto-informante), puesto que parte del corpus está compuesto por textos donde se manifiesta un énfasis en la persona del antropólogo (quien está omitido en el discurso científico tradicional), que vive la experiencia de trabajo de campo etnográfico o reflexiona sobre su disciplina, sin exponer la relación con otro: pero este se deduce en el texto como una "proyección", es decir, como un presencia que ha estado y cuya relación conduce al sujeto antropólogo a buscar un nuevo discurso para referirse a su quehacer profesional.

\section{CONCLUSIÓN}

En conclusión, se justifica la hipótesis presentada: La literatura antropológica en Chile corresponde a un tipo de literatura intercultural en cuanto el sujeto antropólogo/arqueólogo presenta una mirada intercultural de la experiencia de trabajo de campo que supera a la otredad; esta experiencia intercultural se presenta en la medida en que el sujeto se relaciona con el otro desde su integridad, no solo profesionalmente, experimentando una situación intercultural que debido a su fuerza intelectual y sensitiva requiere de estrategias interdisciplinarias, antropológicas y literarias, para comunicarse.

Se justifica a través del análisis del corpus compuesto por 39 textos de 21 antropólogos o arqueólogos chilenos contemporáneos, a partir del desarrollo de los tres objetivos específicos del estudio. En relación con el primer objetivo,

23 En un $48,7 \%$ de los textos se presenta claramente, en un $15,3 \%$ parcialmente y en un $35,8 \%$ no se presenta este aspecto. 
"identificar y analizar los marcos discursivos en relación a la interculturalidad e interdisciplinariedad", se concluye que tanto interdisciplinariedad como interculturalidad corresponden a elementos constituyentes de la propuesta de escritura de la literatura antropológica. En cuanto al desarrollo del tercer objetivo, "identificar y especificar las estrategias discursivas propias de la literatura intercultural presente en los textos", se determinan cuatro estrategias propias de la literatura intercultural; en relación con la hipótesis, destaca la estrategia de "dar cuenta de una situación intercultural", la cual se determina por la particularidad de la situación intercultural que se da en el contexto del viaje y/o trabajo de campo antropológico, en donde entran en contacto las personas del antropólogo o arqueólogo y los informantes, y cuya interculturalidad se manifiesta textualmente a través de la interdisciplinariedad, en la medida en que se requiere de la unión de ambas disciplinas (antropológica y literaria) para dar cuenta de la situación de interculturalidad, situación que implica la participación de los sujetos más allá de sus roles de investigador-informante. Por último, el desarrollo del tercer objetivo, "analizar la relación sujeto-informante presente en los textos a partir de los conceptos de otredad e interculturalidad", da cuenta de la superación de la otredad por medio de la interculturalidad: esto, considerando que la otredad corresponde a la mirada del científico tradicional, que se distancia de los otros para mantener la mirada objetiva, aun cuando requiere insertarse en la comunidad para entender la cultura ajena; mientras que la interculturalidad no requiere tal distanciamiento, por cuanto el sujeto se relaciona con otros desde su propia cultura, pero abierto a cuestionar sus propios parámetros culturales y disciplinarios, y abierto a involucrarse a nivel de persona, más allá de su rol profesional; involucramiento que se traduce en una escritura que supera los márgenes de la escritura científica/objetiva, para proyectarse en una escritura que también es literaria/subjetiva.

\section{BIBLIOGRAFÍA}

Alvarado, Miguel. "Introducción a la antropología poética chilena”. Estudios Filológicos 37 (2002): 169-183.

"La antropología poética chilena como textualidad híbrida". Tesis Doctoral. Universidad Austral de Chile, 2001.

Augé, Marc. El sentido de los otros. Barcelona: Paidós, 1996.

Carrasco, Iván. "Poesía antropológica de Ivonne Valenzuela". Anales de Literatura Chilena 17 (2012): 219-236. 
“Literatura antropológica chilena: fundamentos". Estudios Filológicos 46 (2010): 9-23. "Literatura intercultural chilena: proyectos actuales". Revista Chilena de Literatura 66 (2005): 63-84.

"Interdisciplinariedad, interculturalidad y canon en la poesía chilena e hispanoamericana actual”. Estudios Filológicos 37 (2002): 199-210.

Eco, Umberto. Los límites de la interpretación. Barcelona: Lumen, 1992.

Tratado de semiótica general. Barcelona: Lumen, 1995.

Seis paseos por los bosques narrativos. Barcelona: Lumen, 1996.

Olivares, Juan Carlos. El umbral roto. Escritos en antropología poética. Santiago de Chile: Fondo Matta / Museo Chileno de Arte Precolombino, 1995.

Piña, Carlos. Crónicas de la otra ciudad. Santiago de Chile: FLACSO, 1987.

Recasens, Andrés. “¿Antropología poética o poesía antropológica?”. Primer Encuentro Nacional de Antropología Poética. Ancud-Chiloé, 1988 (sin publicar).

Valenzuela, Pilar. "Interdisciplinariedad e interculturalidad en Metales Pesados de Yanko González Cangas”. Acta Literaria (en prensa).

“Literatura antropológica en Chile: ¿Una literatura intercultural?”. Estudios Filológicos 56 (2015): 161-173.

"Fotografía, literatura y antropología en Atacameños del Siglo XX". Literatura y Lingüística 32 (2015): 99-116.

"El diario de viaje/campo como literatura antropológica en Chile". Tesis Doctoral. Universidad Austral de Chile, 2013.

"Entre antropología y literatura: Recepción de El umbral roto. Escritos en antropología poética". Acta Literaria 45 (2012): 137-151.

\section{ANEXO: CORPUS DE ESTUDIO}

Adán, Leonor. "El etnógrafo invisible". Diarios de campo / de viaje. Ed. Daniel Quiroz. Santiago de Chile: Fondo Matta / Museo Chileno de Arte Precolombino, 2001. 27-32.

Gahona, Alfredo. "Trabajos dispersos de la altura y la sierra: dejos de collas pirquineros". Etnografias mínimas. Ed. Daniel Quiroz. Santiago de Chile: edición personal (Andros Impresores), 2007. 47-50.

"Viaje inesperado al África: De Chiloé a Nairobi”. Diarios de campo / de viaje. Ed. Daniel Quiroz. Santiago de Chile: Fondo Matta / Museo Chileno de Arte Precolombino, 2001. 57-72.

Gallardo, Francisco. "El camino de las almas y el paraíso wayuu”. Etnografías mínimas. Ed. Daniel Quiroz. Santiago de Chile: edición personal (Andros Impresores), 2007. 19-24.

"Notas para una cartografía de la muerte: Toconce 9-16 de junio [diario de campo]". Diarios de campo / de viaje. Ed. Daniel Quiroz. Santiago de Chile: Fondo Matta / Museo Chileno de Arte Precolombino, 2001. 45-56. Arqueología y poesía. Santiago de Chile: LOM, 1997. 
Antropología. Cruzando a través de. Santiago de Chile: Fondo Matta / Museo Chileno de Arte Precolombino, 1995.

González, Yanko. Alto Volta. Valdivia: Ediciones El Kultrún, 2007.

Metales Pesados. Valdivia: Ediciones El Kultrún, 1998.

Jeria, Yuri. "Conversaciones en las costas de Arauco". Diarios de campo / de viaje. Ed. Daniel Quiroz. Santiago de Chile: Fondo Matta / Museo Chileno de Arte Precolombino, 2001. 83-90.

Massone, Mauricio. El rumbo secreto de las ballenas. Punta Arenas: Ediciones Universidad de Magallanes, 2012.

${ }_{2005 .}$ Santa María. Una isla en el desván del tiempo. Concepción: Ediciones Escaparate, "El otro diario de un arqueólogo [fragmentos]". Diarios de campo / de viaje. Ed. Daniel Quiroz. Santiago de Chile: Fondo Matta / Museo Chileno de Arte Precolombino, 2001. 123-138.

Mege, Pedro. La imaginación araucana. Santiago de Chile: LOM Ediciones, 1997.

Mercado, Claudio y Luis Galdames. De todo el universo entero. Santiago de Chile: Fondo Matta / Museo Chileno de Arte Precolombino, 1997.

Montecino, Sonia y Michèle Sarde. La mano de Marguerite Yourcenar. Cocina, escritura y biografia. Cuaderno de recetas (1950-1987). Santiago de Chile: Catalonia, 2014.

Montecino, Sonia. Fuego, hornos y donaciones. Alimentación y cultura en Rapa Nui. Santiago de Chile: Catalonia, 2009.

La olla deleitosa. Cocinas mestizas en Chile. Santiago de Chile: Catalonia, 2005.

Sueño con menguante (Biografía de una machi). Santiago de Chile: Editorial Sudamericana, 1999.

La revuelta. Santiago de Chile: Las Ediciones del Ornitorrinco, 1988.

Olivares, Juan Carlos. "Shamanes en la garúa: antropología poética del Jesús Nazareno de Isla Caguach, Arquipiélago de Chiloé”. Etnografias mínimas. Ed. Daniel Quiroz. Santiago de Chile: edición personal (Andros Impresores), 2007. 25-32.

"Patagoniatango: Crónica de una expedición a la vastedad [antropólogo en viaje]". Diarios de campo / de viaje. Ed. Daniel Quiroz. Santiago de Chile: Fondo Matta / Museo Chileno de Arte Precolombino, 2001. 91-96.

El umbral roto. Escritos en antropología poética. Santiago de Chile: Fondo Matta / Museo Chileno de Arte Precolombino, 1995.

Osorio, Mauricio. Antiguas historias del valle Simpson. Santiago de Chile: Ediciones Nire Negro, 2014.

"Diarios de Melinka". Etnografias mínimas. Ed. Daniel Quiroz. Santiago de Chile: edición personal (Andros Impresores), 2007. 87-90.

Piña, Carlos. Crónicas de la otra ciudad. Santiago de Chile: FLACSO, 1987.

Prieto, Rafael. "Posibilidades de olvidar Puerto Edén”. Diarios de campo / de viaje. Ed. Daniel Quiroz. Santiago de Chile: Fondo Matta / Museo Chileno de Arte Precolombino, 2001. 105-122. 
Quiroz, Daniel. "Diarios de campo/diario de viaje: la vida es un viaje [a modo de introducción]". Diarios de campo / de viaje. Ed. Daniel Quiroz. Santiago de Chile: Fondo Matta / Museo Chileno de Arte Precolombino, 2001. 9-22.

"Olvidada línea del sur y la ruta nacional 40 [sobre trenes y caminos]". Diarios de campo / de viaje. Ed. Daniel Quiroz. Santiago de Chile: Fondo Matta / Museo Chileno de Arte Precolombino, 2001. 97-104.

Ramírez, Dagoberto. “Cuando se diluye el rapport: ¿Una etnografía del fracaso?”. Etnografías mínimas. Ed. Daniel Quiroz. Santiago de Chile: edición personal (Andros Impresores), 2007. 123-132.

Recasens, Andrés. Pueblos de Mar. Relatos etnográficos. Santiago de Chile: Ediciones Departamento de Antropología, Facultad de Ciencias Sociales, Universidad de Chile, 2003. "Balada para niño y perro". 1975. Actas Primer Encuentro Nacional de Antropología Poética. Antropología, Representación, Poética. Ancud: s.e., 1998.

Riedemann, Clemente. Karra Maw'n. Valdivia: Editorial Alborada, 1984.

Rojas, Alexis. "Relato de un naufragio". Etnografias mínimas. Ed. Daniel Quiroz. Santiago de Chile: edición personal (Andros Impresores), 2007. 117-122.

Toledo, Patricio. "En busca del mapuche profundo". Etnografias mínimas. Ed. Daniel Quiroz. Santiago de Chile: edición personal (Andros Impresores), 2007. 57-62.

"La doble ruta antigua: Diario de terreno, Turi, abril-mayo 1993". Diarios de campo / de viaje. Ed. Daniel Quiroz. Santiago de Chile: Fondo Matta / Museo Chileno de Arte Precolombino, 2001. 33-44.

Valenzuela, Ivonne y Juan Pablo Loo. Gracias por el favor concedido. Las animitas de Evaristo Montt, Elvira Guillén y Juana Guajardo. Antofagasta: edición particular (Imprenta Ercilla), 2008.

Atacameños del siglo XX. Registro fotográfico y etnográfico. Antofagasta: edición particular / FONDART, 1998.

Vilches, Flora. "Un verano naranja: Historias de Nueva York". Diarios de campo / de viaje. Ed. Daniel Quiroz. Santiago de Chile: Fondo Matta / Museo Chileno de Arte Precolombino, 2001. 73-82. 